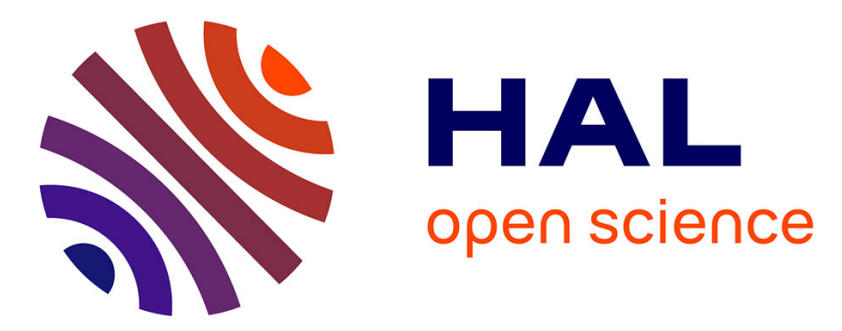

\title{
Charge Transfer Kinetics at Ag(111) Single Crystal Electrode/Ionic Liquid Interfaces: Dependence on the Cation Alkyl Side Chain Length
}

M. Li, S. Liu, L. Xie, J. Yan, Corinne Lagrost, S. Wang, G. Feng, Philippe Hapiot, B. Mao

\section{To cite this version:}

M. Li, S. Liu, L. Xie, J. Yan, Corinne Lagrost, et al.. Charge Transfer Kinetics at Ag(111) Single Crystal Electrode/Ionic Liquid Interfaces: Dependence on the Cation Alkyl Side Chain Length. ChemElectroChem, 2021, 8 (5), pp.983-990. 10.1002/celc.202100094 . hal-03196124

\section{HAL Id: hal-03196124 https://hal.science/hal-03196124}

Submitted on 16 Apr 2021

HAL is a multi-disciplinary open access archive for the deposit and dissemination of scientific research documents, whether they are published or not. The documents may come from teaching and research institutions in France or abroad, or from public or private research centers.
L'archive ouverte pluridisciplinaire HAL, est destinée au dépôt et à la diffusion de documents scientifiques de niveau recherche, publiés ou non, émanant des établissements d'enseignement et de recherche français ou étrangers, des laboratoires publics ou privés. 


\title{
Charge Transfer Kinetics at Ag(111) Single Crystal Electrode/lonic Liquid Interfaces: Dependence on the Cation Alkyl Side Chain Length
}

\author{
Miangang Li ${ }^{[a] \S}$, Shuai Liu ${ }^{[a] \S}$, Liqiang Xie ${ }^{[a]}$, Jiawei Yan ${ }^{[a]^{*}}$, Corinne Lagrost ${ }^{[b]}$, Shuai Wang ${ }^{[c]}$, Guang \\ Feng $^{[c]}$, Philippe Hapiot ${ }^{[\mathrm{b}]^{*}}$, Bingwei Mao ${ }^{[a]^{*}}$
}

In Memoriam: Prof. Jean-Michel Savéant

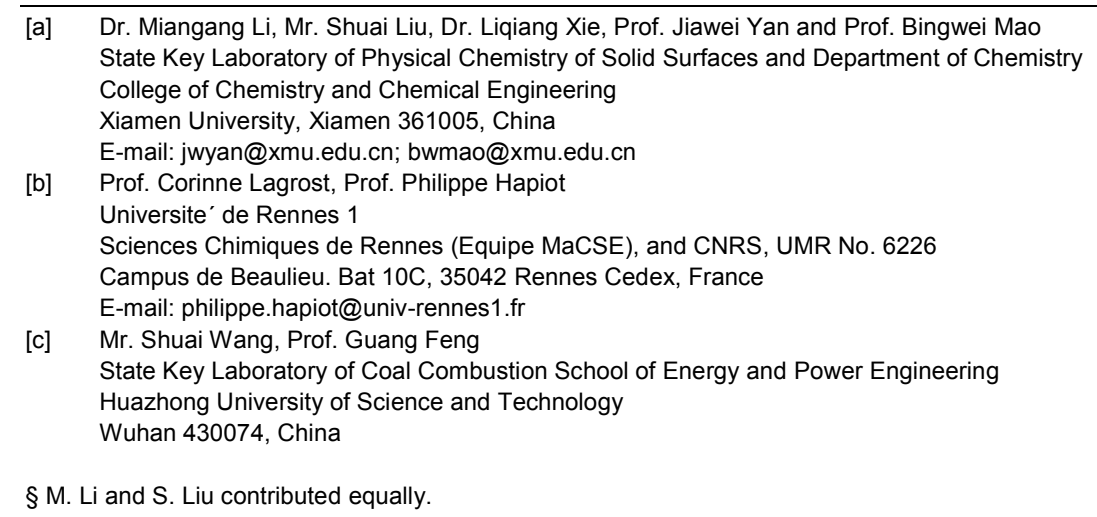

Supporting information for this article is given via a link at the end of the document.

\begin{abstract}
The influences of the electrode/ionic liquids interfaces on the charge transfer kinetics are addressed with the example of the oxidation of chromocene at a single crystal $\mathrm{Ag}(111)$ electrode. We considered three different imidazolium-based room temperature ionic liquids (RTILs) with alkyl chains of different lengths but with a common anion. The standard charge transfer rate constants $k^{\circ}$ are extracted from cyclic voltammograms (CVs) by analyzing the peak potential variations with the scan rate and/or performing semi-integral electroanalysis with a careful treatment of the ohmic drop. Linear variations between the logarithms of $k^{\circ}$ and the longitudinal relaxation times $\pi_{\llcorner}$or the dynamic viscosity $\eta$ of the ionic liquids are obtained. However, the amplitude of the decrease is much larger than it could be expected from the sole bulk properties of the ionic liquid indicating a strong effect of the interface on the kinetics. These observations agree with a well-organized electrode/RTIL interface that behaves as a sort of self-assembled monolayer that controls the charge transfer kinetics with an exponential tunneling coefficient $\beta$ of $5.51 \mathrm{~nm}^{-1}$.
\end{abstract}

\section{Introduction}

Room-temperature ionic liquids (RTILs) are a class of solvent consisting entirely of ionic species and being fluid at or around room temperature. In the last two decades, they have attracted extensive interests due to their wide electrochemical windows, low vapor pressure and good ionic conductivity. ${ }^{[1]}$ RTILs are considered as designer solvents since their properties could be modulated through possible combinations of numerous cations and anions. However, unlike the extensive applications of RTILs in electrochemistry, studies on both the structure of electric double layer (EDL) and charge transfer kinetics at electrode/ionic liquid interfaces are still the subject of numerous questions..$^{[1 \mathrm{~d}, 1 f}$ 19] In contrast to conventional electrolytes, the concentration of ions near the electrode surface in ionic liquids remains to be high as in bulk resulting in special effects regarding the electrode/RTIL interface organization. ${ }^{[1 \mathrm{~d}, 1 \mathrm{f}, 1 \mathrm{~g}]}$ For example, it has been shown that ions of RTIL such as imidazolium-based cations can strongly adsorb at Au single crystalline electrode surfaces forming wormlike ${ }^{[2]}$ and micelle-like ${ }^{[2 d, 3]}$ structures on the (111) and (100) planes, respectively, while they form layered structures near the surface, $[2 b, 4]$ which were also predicted by theoretical simulations. [5] Furthermore, Kornyshev et al. developed a simple continuum theory to describe the EDL structure of electrode/RTIL interface. [6] An "overscreening" structure is suggested to form at moderate potential with respect to the potential of zero charge (PZC), while the structure transforms to "crowding" as the potential shifts to rather high values. In the overscreening regime, the first layer near the electrode provides more counter-charges than are present on the surface resulting in oscillations in the potential profile that are gradually overcome at higher potential leading to an exponential profile of the potential. ${ }^{[1 d, 1 h, 5 b, 7]}$ The lateral and vertical structures and resultant potential distributions in the ionic liquid side of the EDL of interfaces are indeed distinctive from those in classical electrolyte systems. 
Heterogeneous charge transfer reactions are important processes in fundamental and applied electrochemistry ${ }_{,}^{[8]}$ the kinetics of which, such as the electron transfer across an electrode/electrolyte interface, is strongly dependent upon the solvent nature and the interfacial structures. Consequently, numerous studies have been dedicated to the investigations of electron transfer kinetics in RTILs in the past two decades. ${ }^{[9]}$ As a general tendency, charge transfer processes were found to be slower in ionic liquids than in classical molecular solvents. ${ }^{[1 \mathrm{c}, 1 \mathrm{e}, 11 \text {, }}$ $1 \mathrm{~m}, 1 \mathrm{n}]$ This was mainly ascribed to larger solvent reorganization energies in the solvents with high ion concentrations or lower relaxation time in RTILs. However, while theoretical models of interfacial charge transfer kinetics in RTILs are scarce, experimental works were mainly performed using polycrystalline electrodes at which the EDL structures are not well-defined, making more difficult characterizing the effects of the interface on the charge transfer kinetics. ${ }^{[9]}$ Therefore, a basic understanding of the correlation between the charge transfer kinetics and the structure at electrode/RTIL interfaces is still lacking and for this it is essential to utilize single crystalline electrodes as substrates for further investigations of both EDL and its influence on interfacial charge transfer kinetics. ${ }^{[10]}$

In this study, we use an $\mathrm{Ag}(111)$ single crystal electrode to study the charge transfer kinetics and its correlation with the EDL structure of electrode/RTIL interfaces. We focus on the standard charge transfer kinetics in three RTILs composed of the same bis(trifluoromethanesulfonyl)imide (TFSI) anions associated to imidazolium cations with different lengths of their alkyl side chains. $\mathrm{Ag}(111)$ was chosen as the working electrode, because it undergoes no surface reconstruction, cation etching or specific adsorption in all the three RTILs as we reported before ${ }^{[4 d]}$. In addition, the Ag single crystal surface can be made sufficiently small to facilitate fast voltammetry measurements. Noteworthy is that chemical polishing is adequate for obtaining well-defined surfaces of Ag electrodes, without flame annealing procedure and hanging meniscus strategy like for Au single crystal electrodes which prevent the use of small electrodes. We choose chromocene (Cc), a metallocene that is analogue of ferrocene, as a probe in this study since its formal potential locates in the potential range where $\mathrm{Ag}$ remains unoxidized in a range of potential more negative than the PZC. The chemically reversible one-electron oxidation of $\mathrm{Cc} \leftrightarrow \mathrm{Cc}^{+}+e^{-}$can be regarded as a fast outer-sphere process as in conventional electrolytes. ${ }^{[11]}$

\section{Results and Discussion}

\section{Charge transfer rate constant $k^{\circ}-$ Variation of $\Delta E_{\mathrm{p}}$ with the scan rate.}

Cyclic voltammetry (CV) is a widely employed electrochemical technique for investigating the charge transfer kinetics and was used with success for evaluating electron transfer rate constants in RTILs. ${ }^{[1 c, 9 d, 9 e, 12]}$ Voltammograms of the Cc oxidation in the different RTILs are provided on Figure 1 displaying well-resolved CVs in the range of investigated scan rates. Peak-to-peak potential separation $\Delta E_{\mathrm{p}}$ increases with scan rate $v$ within the high scan rate range in all the three RTILs and this increase is larger for experiments in the RTILs with the longer alkyl chains cations at a given scan rate.
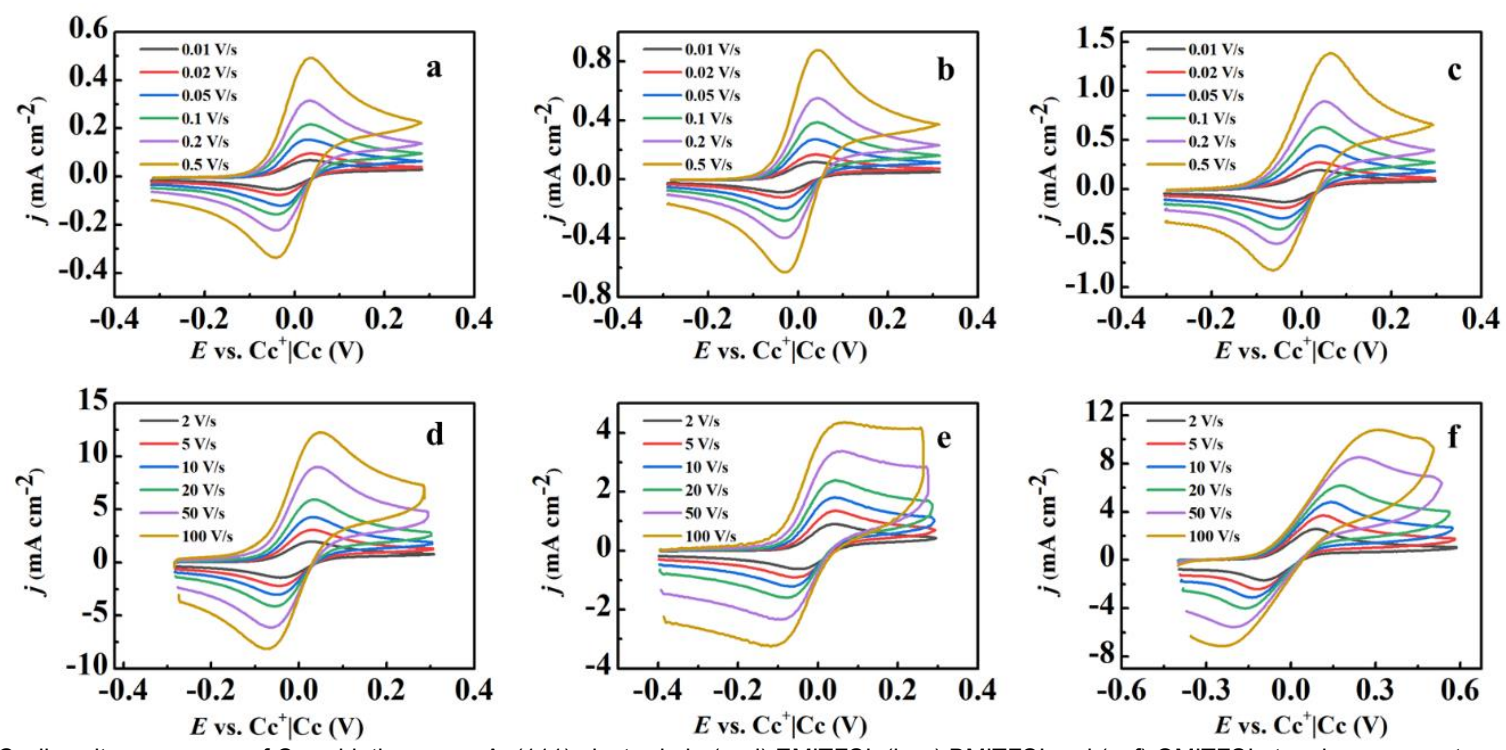

Figure 1. Cyclic voltammograms of Cc oxidation on an Ag(111) electrode in (a, d) EMITFSI, (b, e) BMITFSI and (c, f) OMITFSI at various scan rates ranging from 0.01 to $100 \mathrm{~V} \mathrm{~s}^{-1}$. Ohmic drop and capacitive current were corrected after measurements (see Supporting Information for details).

Assuming that Butler-Volmer law prevails, $k^{\circ}$ values could be derived from the relationship between the peak-to-peak potential separation between the anodic and cathodic processes, $\Delta E_{\mathrm{p}}$, and scan rate $v$. Figure 2 depicts the variations of $\Delta E_{\mathrm{p}}$ with $\log (v)$ in the three systems. There are several advantages of considering $\Delta E_{\mathrm{p}}$ for such determination, notably the quantity is insensitive to the reference electrode potential shift that may occur during the set of experiments. ${ }^{[8 a, 12]}$
The electrochemical behavior and the variation of $\Delta E_{\mathrm{p}}$ shown in Figure 2 depend on a single dimensionless parameter $\Lambda=\frac{k^{\circ}}{\sqrt{D}} \sqrt{\frac{R T}{\alpha F v}}$ that combines the standard rate constant $k^{\circ}$ with the transfer coefficient $\alpha$, diffusion coefficient $D$ of chromocene. ${ }^{[8 a]}$ Here, $\Lambda$ can be used to estimate the transition between reversible and irreversible electrochemical reactions. At low scan rates typically for $\Lambda \gg>0.1$, the electrochemical reaction appears 
reversible and $\Delta E_{\mathrm{p}}$ remains unchanged; while at high scan rates, quasi-reversible and even irreversible characters for the highest scan rates appear (typically for $\Lambda<<0.1$ ), and $\Delta E_{\mathrm{p}}$ increases with $v$. In the quasi-reversible region, the shape of the variation of $\Delta E_{\mathrm{p}}$ is almost insensitive to the transfer coefficient and permits an easy determination of $k^{\circ}$ since the variation depends only on one parameter $\Lambda$. Interestingly, it can be seen on Figure 2 that the longer the cation alkyl side chain is, the lower is the scan rate at which the reaction enters irreversible regime, indicating a lower charge transfer rate constant of $\mathrm{Cc}$ in the ionic liquid with a longer cation alkyl chain.
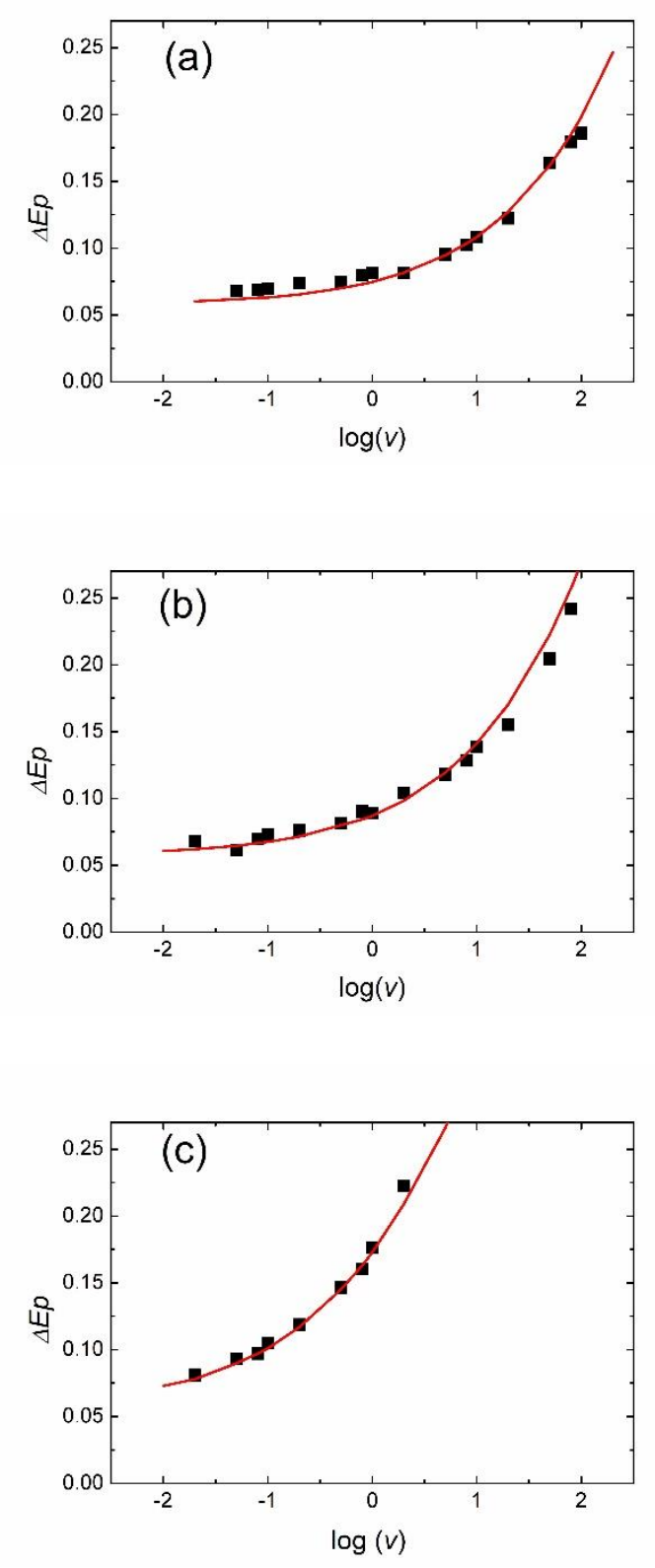

Figure 2. Anodic and cathodic peak potential separations $\Delta E_{p}$ in $\mathrm{V}$, taking into account the ohmic drop, as function of logarithmic scan rate $v$ in $V \mathrm{~s}^{-1}$ for the oxidation of $\mathrm{Cc}$ in (a) EMITFSI, (b) BMITFSI and (c) OMITFSI. Lines are the theoretical behaviors for $k^{\circ}=4.0 \times 10^{-2}, 1.0 \times 10^{-2}, 2.7 \times 10^{-4} \mathrm{~cm} \mathrm{~s}^{-1}$. (log is the decimal logarithm). Errors on the determined $k^{\circ}$ are around $\pm 20 \%$.

Simulation of the voltammograms without removal of ohmic drop and capacitive current was performed to determine the values of $k^{\circ}$. However, the solution resistance $R_{\mathrm{s}}$ and double layer capacitance $C_{d}$ could make severe distortions to the voltammograms at high scan rates. [8a, 12] Especially, uncompensated $R_{\mathrm{s}}$ affects the variation between $\Delta E_{\mathrm{p}}$ and $v$ and renders difficult an accurate determination of $k^{\circ}$. We choose a post-treatment procedure that was introduced by Savéant et al. in the 70's in which the simulated variations of $\Delta E_{\mathrm{p}}$ are calculated taking into account the ohmic drop (See the Supporting Information Part for details ${ }^{[13]}$. The fitting of the experimental points with theoretical curves provides the parameter $\frac{k^{\circ}}{\sqrt{D}}$ and then the values of $k^{\circ}$. Here the diffusion coefficients $D$ of Cc were determined by recording cyclic voltammograms in steady state regime on a microelectrode and in the transient state regime on a millimeter diameter disk electrode in the same solution[14a, 14b] (See the Supporting Information Section for details). ${ }^{[14 c]}$ The derived diffusion coefficients of $\mathrm{Cc}$ in EMITFSI, BMITFSI and OMITFSI were found to be $5.7 \times 10^{-7}, 3.2 \times 10^{-7}$ and $9.5 \times 10^{-9}$ $\mathrm{cm}^{2} \mathrm{~s}^{-1}$, respectively, in agreement with literature for similar metallocene analogues in RTILs. ${ }^{[15]}$ We mention that it is more appropriate to consider the measured $D$ as apparent diffusion coefficients since the nature of the diffusion process in RTILs is still the subject of controversy. It is noticeable that good agreements are obtained between the experimental data and the theoretical curves (lines on Figure 2) when using the values of $R_{\mathrm{s}}$ and $C_{\mathrm{d}}$ that were independently measured by EIS. From the fits, we measured the $k^{\circ}$ values of $\mathrm{Cc}$ in the three different ionic liquids (see Table 1). Given a $k^{\circ}$ value equal to $0.04 \mathrm{~cm} \mathrm{~s}^{-1}$ in EMITFSI, the increase of side-chain length leads to reduced values of $k^{\circ}$ by around 4 times in BMITFSI and around 150 times in OMITFSI, showing a significant impact of the cation alkyl chain length on the electron transfer kinetics.

\section{Evaluation of the transfer coefficients $\alpha$ and standard rate constants $\mathrm{k}^{\circ}$ by semi-integral (convolution) analysis.}

The semi-integral analysis provides a direct view of the relationship between current and potential including charge transfer kinetic parameter without the pre-assumption of a model and complements the previous approach. $\left.{ }^{[8 a}, 16\right]$ The method was notably used to demonstrate that the transfer coefficient varies with the potential and the occurrence of an $i-E$ quadratic law (Marcus Model). The main aspect of semi-integral analysis, also referred as convolutive voltammetry, is the calculation of the semiintegral current $l(t)$, as follows: ${ }^{[16 \mathrm{~b}]}$

$$
I(t)=\frac{1}{\pi^{1 / 2}} \int_{0}^{t} \frac{i(u)}{(t-u)^{1 / 2}} d u
$$

Briefly, semi-integral converts a CV to an S-shaped semi-integral voltammogram of $I(t)$ versus $E$ relationship. In the case of an irreversible CV (typically when $\Lambda<<0.1$ ), for a mono-electron process, the charge transfer rate constant $k^{0}$ can be determined from the following equation: ${ }^{[16 b]}$

$$
\ln \left(\frac{I_{l}-I(t)}{i(t)}\right)=-\frac{\alpha F}{R T}\left(E-E^{0}\right)-\ln \frac{k^{0}}{D_{R}^{1 / 2}}
$$

$E^{0}$ is the formal potential which is taken as the midpoint of the anodic and cathodic peak potentials of the $\mathrm{CV}$ at the lowest possible scan rate. Notice that the validity of such analysis by equation (2) requires that the charge transfer should be the limiting step. Considering the highest available scan rate in our study (around $100 \mathrm{~V} \mathrm{~s}^{-1}$ ), we evaluate the reversibility character by examining the parameter $\Lambda$ in the three ionic liquids. The scan rates available for experiments in the three RTILs are mainly within the quasi-reversible regime and experiments are not adapted for a determination of the transfer coefficient $\alpha$. 
Considering the previously determined $k^{\circ}$ and taking $\alpha$ values around 0.5 , at $v=100 \mathrm{~V} \mathrm{~s}^{-1}$, we obtained $\Lambda=0.84,0.28$, and 0.043 for EMITFSI, BMITFSI, and OMITFSI, respectively, confirming that $\alpha$ could only be determined at the highest scan rate in OMITFSI, just estimated for BMITFSI and unfortunately not reachable for EMITFSI simply because the irreversible regime is outside our experimental window.

Figure 3 shows the semi-integral voltammograms translated from the $\mathrm{CV}$ of Figure 1 recorded at a scan rate of $100 \mathrm{~V} \mathrm{~s}^{-1}$ for
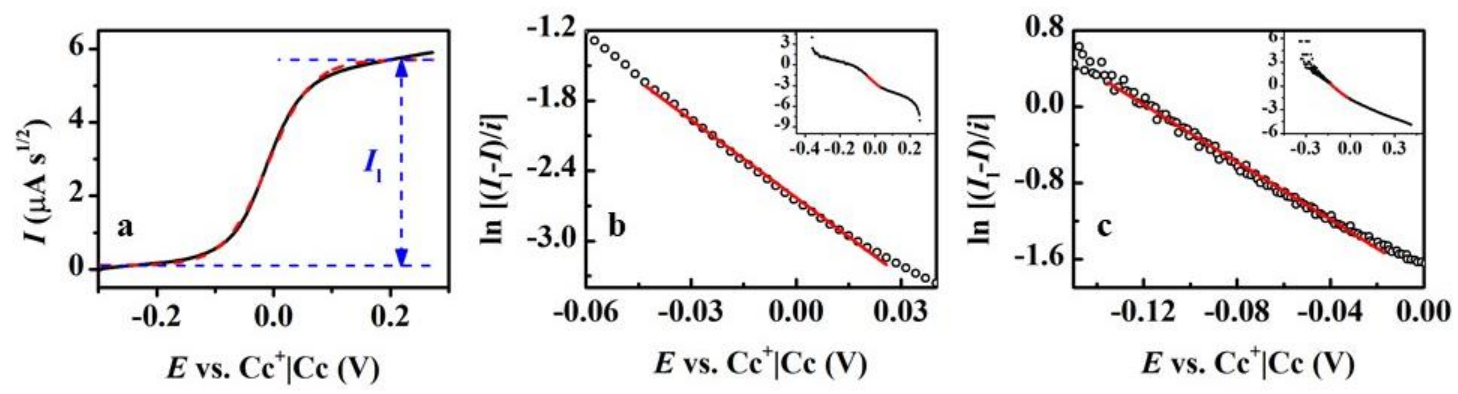

Figure 3 (a) The procedure for determining $h$ of a semi-integral voltammogram from an irreversible process. The black solid line is the semi-integral voltammogram and the red dashed line is its Boltzmann growth fitting. (b-c) Logarithmic analysis of semi-integral current to potential in BMITFSI and OMITFSI respectively. The fitted lines (red) are obtained in the linear regions. The whole plots are presented in the insets.
As seen in Table 1, a reasonable agreement is obtained for the $k^{\circ}$ values considering the uncertainties for the two methods. Notably, contrary to the simulation method based on $\Delta E_{p}$ measurement, the logarithm analysis of equation 2 is sensitive to a possible shift of the reference potential, as it requires an independent measurement of the standard potential $E^{\circ}$. Nevertheless, both values follow the same trend of strong length dependency of cation alkyl side chain with that obtained by simulation. Additionally, transfer coefficient $\alpha$ decreases with the cation side chain lengths when passing from BMITFSI to OMITFSI. However, it must be pointed out that semi-integral electroanalysis is best relevant under the conditions that background current remains constant and solution resistance influence is fully corrected within the potential range of interest. In practice, even if a working electrode with small diameter is used, high solution resistance and variation of the double layer capacitance (even slightly) with the potential in our RTILs ${ }^{[4 d]}$ make difficult to completely mitigate artifact effects especially when faster scan rates are used. However, the $k^{\circ}$ values obtained by semi-integral electroanalysis are in reasonable agreement with those derived from $\Delta E_{\mathrm{p}} / \log (v)$ analysis, which supports the validity of the analyses.

Table 1. Kinetic parameters of Cc oxidation in RTILs obtained by $\Delta E_{\mathrm{p}}$ and semiintegration analyses.

\begin{tabular}{ccccc}
\hline & $\begin{array}{c}\boldsymbol{k}^{\circ} \\
\left(10^{-7}\right. \\
\left.\mathrm{cm}^{2} \mathrm{~s}^{-1}\right)\end{array}$ & $\begin{array}{c}\left(10^{-3} \mathrm{~cm} \mathrm{~s}^{-1},\right. \\
\left.\Delta E_{p} \text { analysis }\right)\end{array}$ & $\begin{array}{c}\boldsymbol{k}^{\circ} \\
\left(10^{-3} \mathrm{~cm} \mathrm{~s}^{-1},\right. \\
\text { Semi-integration })\end{array}$ & $\begin{array}{c}\boldsymbol{\alpha} \\
\text { (Semi- } \\
\text { integration) }\end{array}$ \\
\hline EMITFSI & 5.7 & 40 & - & - \\
BMITFSI & 3.2 & 10 & $7.8^{*}$ & $0.58^{*}$ \\
OMITFSI & 0.095 & 0.27 & 0.59 & 0.39 \\
\hline
\end{tabular}

\section{The dependency of $k^{0}$ on cation alkyl side chain lengths.}

As seen in Table 1, the increase of the cation alkyl chain length of the RTILs results in a considerable decrease of the rate
${ }^{*}$ Estimated (See text)
BMI and OMI-based ionic liquids. The plateau of the S-type is not flat but slightly tilted. Therefore, a Boltzmann growth fitting was applied in the determination of $l_{1}$. The charge transfer rate constant $k^{\circ}$ and $\alpha$ of $\mathrm{Cc}$ are then analyzed based on equation (2). From the plots of the logarithmic function of current versus potential ( $3 \mathrm{~b}$ and $3 \mathrm{c}$ ), the values of $k^{\circ}$ and $\alpha$ are calculated using the diffusion coefficient $D$ obtained in the earlier part of the section. constant $k^{\circ}$. In the framework of the Marcus theory, the standard rate constant corrected by the effect of the double layer and for an adiabatic electron transfer is given by: ${ }^{[8 b, 9 b]}$

$$
k^{\circ}=\frac{K_{p}}{\tau_{L}}\left(\frac{\Delta G_{O s}^{\#}}{4 \pi \mathrm{R} T}\right)^{1 / 2} \exp \left(-\frac{\Delta G_{O s}^{\#}+\Delta G_{i s}^{\#}}{\mathrm{R} T}\right)
$$

where $K_{p}$ is the equilibrium constant for a precursor complex and $\tau_{L}$ is the longitudinal relaxation time that is the relaxation time of the solvent normalized by the ratio of the static $\varepsilon_{s}$ and high frequency $\varepsilon_{o p}$ relative permittivities, $\Delta G_{O s}^{\#}, \Delta G_{i s}^{\#}$ are the standard Gibbs activation energies of the outer sphere and inner sphere contributions, respectively. For large redox molecules in polar solvents, the different terms of the relation are similar and only $\tau_{L}$ significantly varies between solvents. Within these simplifications, it is commonly observed that $k^{\circ}$ and $1 / \tau_{L}$ follow a similar trend for a given redox molecule. ${ }^{[8 b, 9 a, 9 b]}$ Furthermore, using data from literature ${ }^{\left[{ }^{9 b]}\right.} \tau\llcorner$ are estimated to be $6.8,9.0,14.9 \mathrm{ps}$ for EMITFSI, BMITFSI and OMITFSI respectively. A direct correlation between the measured $k^{\circ}$ and $\tau$ is presented in Figure 4. The black lines on Figures 4 illustrate the theoretical behaviors expected for a linear variation considering that such lines pass through the origin. It is noticeable that $k^{\circ}$ decreases more rapidly than expected for a linear variation with $1 / \tau$.

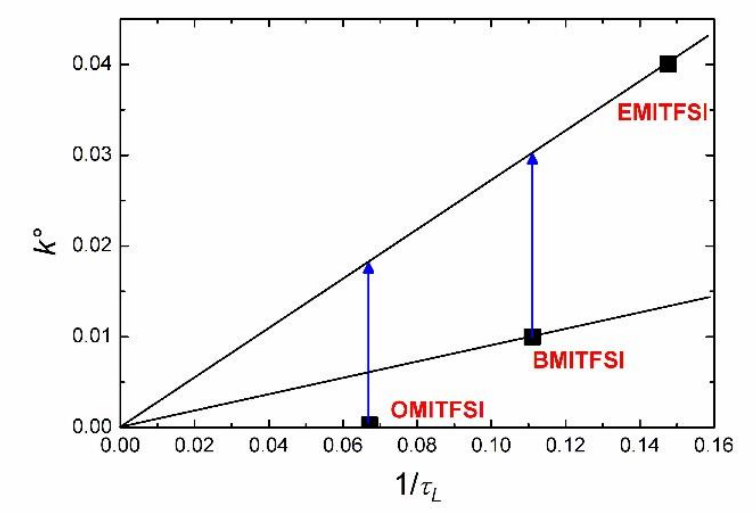


Figure 4. Variations of $k^{\circ}$ in $\mathrm{cm} \mathrm{s}^{-1}$ with $1 / \tau_{L}$ in $\mathrm{ps}^{-1}$. Lines are the variations expected from a linear variation between $k^{\circ}$ and $1 / \tau_{L}$. Blue arrows show the estimations of $k_{t}$ considering EMITFSI as reference (see text).

Another way of illustrating such a dependency is to consider a plot of $\ln \left(k^{\circ}\right)$ versus $\ln (\tau L)$ that is expected to present a slope of 1 for an adiabatic process if the relationship between $k^{\circ}$ and $1 / \tau$ L was followed. $\tau_{L}$ was estimated from dielectric spectroscopy measurements ${ }^{[17]}$ and according to the proposed method of calculating a harmonic average relaxation time..$^{[9 b, 17]}$ As seen on Figure 5, a linear plot is indeed obtained but with a slope of -6.45 that is obviously more intense than the expected -1 value.

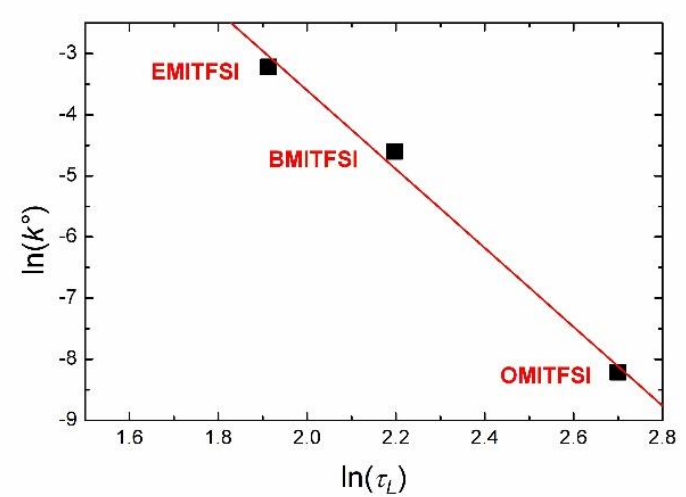

Figure 5. Variations of $\ln \left(k^{\circ}\right)$ in $\mathrm{cm} \mathrm{s}^{-1}$ with the longitudinal relaxation time $\tau_{L}$ in ps. Line is the fit for the linear variation (slope: -6.45 ). Error on $\ln \left(k^{\circ}\right)$ is estimated as lower than \pm 0.2 .

Additionally, according to the Stokes-Einstein-Debye equation, because $\tau_{L}$ correlates with the dynamic viscosity $\eta$, in many publications, correlations between $k^{\circ}$ and $\eta$ were often reported especially when measurements of $\tau_{\llcorner}$were not available. ${ }^{[8 \mathrm{~b}, 9 \mathrm{9a}]}$ For example in relation with the current work, Bentley et al. reported a variation showing that the logarithmic $k^{0}$ for ferrocene oxidation in a series of RTILs is proportional to the logarithmic viscosity $\eta$ with a slope close to the expected $-1 .{ }^{[9 a]}$ The logarithmic variations of $k^{\circ}$ with $\eta$ for our experiments are shown on Figure 6 . A linear variation is undeniably obtained but with a slope of -5.07 confirming a much faster decrease of $k^{\circ}$ than the expected -1 slope for a change of viscosity or $\tau_{L}$ of the RTILs.

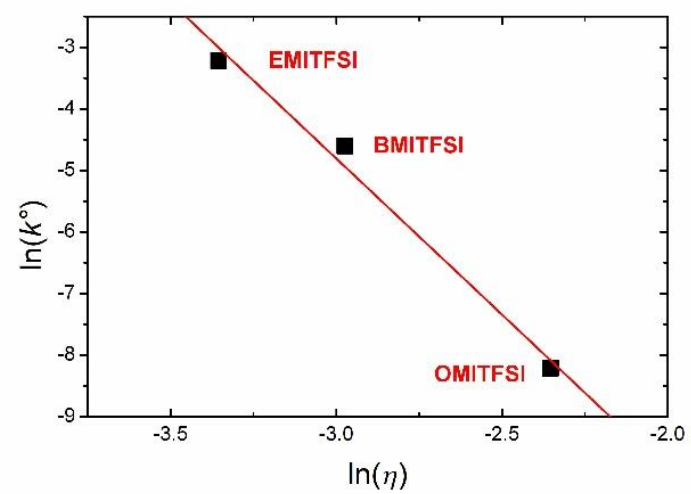

Figure 6. Variation of $\ln \left(k^{\circ}\right)$ in $\mathrm{cm} \mathrm{s}^{-1}$ with the viscosity of the ionic liquid in Pa.s Line is the fit for the linear variation (slope: -5.07$)$. Error on $\ln \left(k^{\circ}\right)$ is estimated as lower than \pm 0.2 . Viscosity values of the three ionic liquids are taken from ref. [17].
Notice that the quoted experiments were performed with RTILs with cations having similar sizes and on a polycrystalline $\mathrm{Pt}$ electrode ${ }^{[9 a]}$ contrary to our experiments performed with a single crystal electrode. This probably accounts for the difference of observed behaviors. As explained in the introduction, it is very likely that the EDL structure, which is not considered in the above equations, plays an important role in the reaction kinetics in RTILs and could explain the amplitude of the variations.

Based on the information gained from in-situ STM and AFM force curve measurements, strong adsorption of RTILs is not observed on a negatively charged $\mathrm{Ag}(111)$ electrode surface, while a layered structure is formed at $\mathrm{Ag}(111) / \mathrm{EMITFSI}$ interface ${ }^{[4 \mathrm{~d}]}$ and the $\mathrm{EMI}^{+}$cations are located closer to the electrode surface in a vertical configuration. Similar organizations were also observed for $\mathrm{BMI}^{+}$or $\mathrm{HMI}^{+}$(1-hexyl-3-methylimidazolium) on $\mathrm{Ag}$ electrode by surface-enhanced Raman spectroscopy. ${ }^{[18]}$

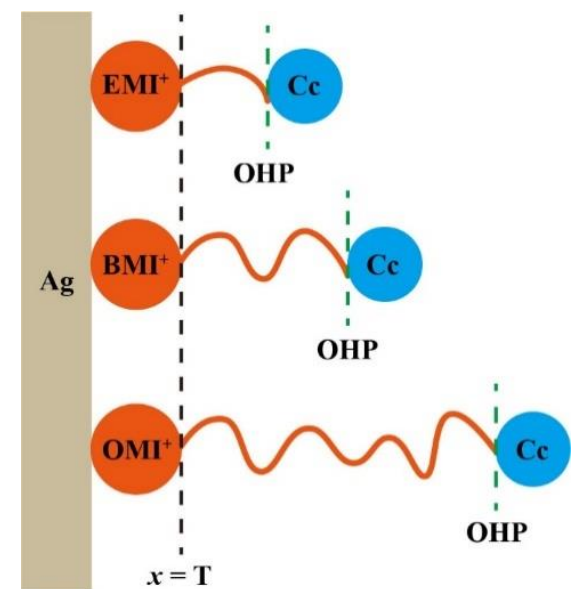

Scheme 1. Schematic illustration of the proposed structure and reaction sites at $\mathrm{Ag}(111) / \mathrm{RTIL}$ interface. Dashed lines indicate the locations of transition state and OHP.

The ion arrangement of the first layer at $\mathrm{Ag}(111) / \mathrm{RTIL}$ interface is proposed as illustrated in Scheme 1. The imidazolium rings vertically adsorb at $\mathrm{Ag}(111)$ with the alkyl side chains extending towards the bulk solution and the anions located next to them Assuming the location where Cc oxidation occurs (i.e. OHP) is at the plane between alkyl side chains and anions, and the transition state of the reactant is located at the junction of imidazolium ring and alkyl side chain (marked as $x=\mathrm{T}$ ), $d$ is the distance from the OHP to the position of the reactive site. In this scheme, the oxidation of $\mathrm{Cc}$ could be seen as similar to an electron tunneling process between an electrode and an electroactive species held at a fixed distance from the electrode surface by an alkyl spacer. An electron tunneling process presents a logarithmic $k^{\circ}$ relationship with tunneling distance $d$ and it is reasonable to suggest that $d$ is the length of cation chain of the studied RTILs: ${ }^{[19]}$

$$
k^{\circ}=k_{t} \exp (-\beta d)
$$

where $\beta$ is denoted as tunneling coefficient and $k_{t}$ hypothetical rate constant that would be the expected $k^{\circ}$ for a "pure" linear variation with $1 / \tau_{\llcorner}$i.e. without the interface screening. To check the validity of this scheme, we could evaluate the attenuation on $k^{\circ}$ normalized by $k_{t}$. Using EMITFSI as the reference RTIL, the analysis consists in plotting the attenuation $\ln \left(k \% k_{t}\right)$ versus the variation of the cation length $d-d_{E M}$ where $k_{t}$ is the value of $k^{\circ}$ extrapolated on the straight line of EMITFSI for the corresponding $1 / \tau_{\llcorner}$(see the blue arrows on Figure 4 ), and $d-d_{E M I}$ the tunneling 
distances calculated from DFT calculations of the cation size. Table 1 summarizes the lengths of the imidazolium rings and whole cations, values of $k^{\circ}$ and $k t$ for the oxidation of $\mathrm{Cc}$ in the three RTILs and the estimated attenuation on $k^{\circ}$ due to the tunneling effect that are plotted on Figure 7.

Table 2. Estimation of the tunneling parameters based on the sizes of the RTIL cations.

\begin{tabular}{cccccc}
\hline & $\begin{array}{c}\text { Imidazoliu } \\
\mathrm{m} \text { ring } \\
(\mathrm{nm})\end{array}$ & $\begin{array}{c}\text { Cation } \\
(\mathrm{nm})^{\mathrm{a}}\end{array}$ & $\begin{array}{c}d-d_{E M I} \\
(\mathrm{~nm})\end{array}$ & $\begin{array}{c}k_{t}\left(10^{-3} \mathrm{~cm}\right. \\
\left.\mathrm{s}^{-1}\right)\end{array}$ & $k \% k_{t}$ \\
\hline EMITFSI & 0.556 & 0.751 & 0 & 40 & 1 \\
BMITFSI & 0.555 & 0.996 & 0.245 & 30.5 & 0.327 \\
OMITFSI & 0.556 & 1.503 & 0.752 & 18.4 & 0.015 \\
\hline
\end{tabular}

a The lengths of imidazolium rings and whole cations are calculated based on the density functional theories by using B3LYP with the $6-311+G(2 d, p)$ basis set.

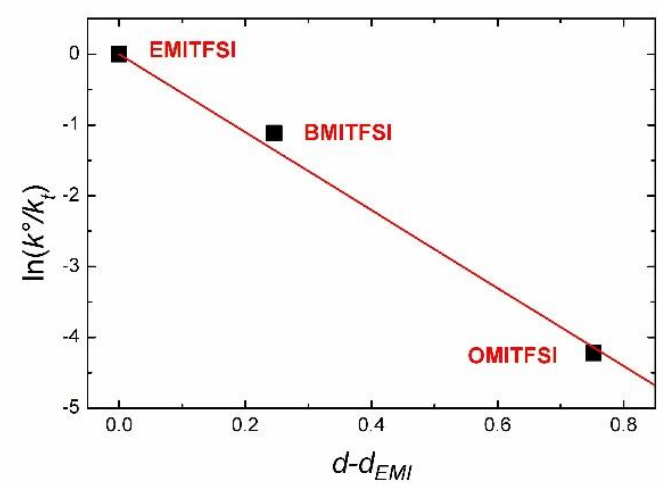

Figure 7. Variation of $\ln \left(k \% k_{t}\right)$ for the oxidation of $\mathrm{Cc}$ with the change of length of the cation $d-d_{E M I}$ in $\mathrm{nm}$ considering the data in EMITFSI as a reference. Line is the linear fit with a slope equal to $-5.51 \mathrm{~nm}^{-1}$.

As seen on Figure 7, a linear variation is obtained with a slope corresponding to an apparent tunneling coefficient $\beta$ of $5.51 \mathrm{~nm}^{-1}$. The tendency of the variation is fully consistent with the structure proposed in Scheme 1 but the value of $\beta$ is notably smaller than the data reported for a compact self-assembled alkyl monolayer (in the range of $8.7-9.0 \mathrm{~nm}^{-1}$ ). ${ }^{[19 a]}$ If the layer is well ordered, it is probably less compact than a strongly attached alkyl layer and Cc could enter inside the layer and approach closer to the electrode surface. Another explanation is that the position of the alkyl chains is not perpendicular to the surface leading to an overestimation of the layer thickness by simply considering $d-d_{E M I}$. In addition, it is unlikely that the cation could totally lay on the surface, but there is probably an angle between the electrode plane and the imidazolium cation. In such a case, the titled orientation of the cation could also contribute to the observation of reduced $\beta$ value. Furthermore, the exponential relationship of the Figure 7 could be discussed in view of the theoretical works related to EDL in RTILs (see the references quoted in $1 d-h$ ) notably about the effects of the "overscreening" and the oscillations of the potential profile on the driving force of the electron transfer. The regular decrease observed on Figure 7 with the size of the cation supports the hypothesis that overscreening does not considerably affect the electrode kinetics. However, the semi-integral analysis indicates that the transfer coefficient $\alpha$ is considerably smaller than 0.5 for OMITFSI and appears decreasing between BMITFSI and OMITFSI. This observation is not elucidated as it clearly differs from the case of an electron transfer through an alkyl selfmonolayer where $\alpha$ is found close to 0.5 and with little variation with the chain length of the cation. ${ }^{[19 a]}$ Impedance spectroscopy have revealed subtle changes in the structure of the $\mathrm{Ag}(111) / \mathrm{RTIL}$ interface with the potential notably in the not very negative potential region where $\mathrm{Cc}$ oxidation occurs but no clear correlation with the present results could be done. ${ }^{[4 \mathrm{~d}]}$ We expect that in the future experiments can be combined with simulations to elucidate the variation of $\alpha$ with the electrode/RTIL interfacial structure in detail.

\section{Conclusions}

The results presented in this study demonstrate the considerable influence of the electrode/RTIL interface on the charge transfer kinetics at an electrode that could be reduced by several orders of magnitude. We observe that this decrease due to the interface varies exponentially with the size of the cation of the RTILs. Considering that the oxidation of chromocene on $\mathrm{Ag}(111)$ occurs in the negative branch of potentials versus the point of zero charge, this observation falls in line with an interface structure where the cations are located close to the electrode surface in a vertical configuration as previously proposed from AFM measurements.

\section{Experimental Section}

1-ethyl-3-methylimidazolium bis(trifluoromethylsulfonyl)imide (EMITFSI),

1-butyl-3-methylimidazolium bis(trifluoromethylsulfonyl)imide (BMITFSI) and 1-octyl-3methylimidazolium bis(trifluoromethylsulfonyl)imide (OMITFSI) were purchased from loLiTec in the highest available quality $(99 \%)$, and were vacuum-dried for several hours at $353 \mathrm{~K}$ to remove the absorbed water and oxygen prior to the use. Solutions of Cc were then prepared by direct addition of Cc powder ( $95 \%$ purity from Aldrich) to ionic liquids. The nominal concentration of Cc in EMITFSI or BMITFSI is around $15 \mathrm{mM}$, and that in OMITFSI is around $100 \mathrm{mM}$. The structures of the RTILs and Cc are illustrated in Scheme 2. All the preparations as well as the electrochemical studies were carried out in a glovebox filled with 99.999\% $\mathrm{Ar}\left(\mathrm{H}_{2} \mathrm{O}\right.$ and $\mathrm{O}_{2}$ levels were less than $\left.1 \mathrm{ppm}\right)$, which limited the degradation of $\mathrm{Cc}$ and solutions were found to be stable during the time of the experiments. 

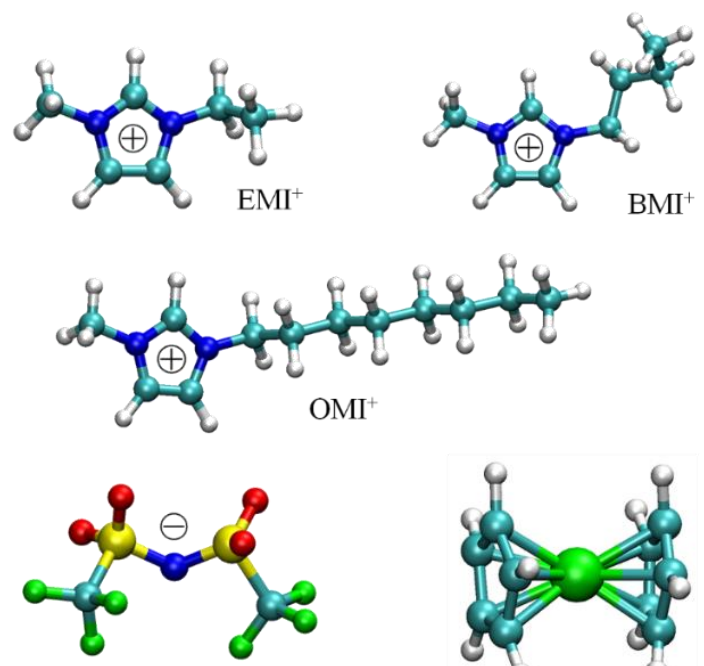

TFSI $^{-}$

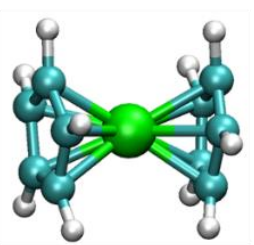

$\mathrm{Cc}$
Scheme 2. Structures of the constituent cations and anion of RTILs and Cc used in this study.

A Ag(111) single crystal rod with diameter of $1 \mathrm{~mm}$ (MaTecK, Germany) was selected as a working electrode for cyclic voltammetry and electrochemical impedance spectroscopy (EIS) experiments. A Ag wire and a Pt wire were used as reference and auxiliary electrodes, respectively. The electrochemical cell and pretreatments of the electrodes are the same as our previous work $^{[4 \mathrm{~d}]}$.

All the electrochemical studies were performed with an Autolab potentiostat (Eco-Chemie, Netherlands) controlled by the Nova 2.0 software package. Cyclic voltammetry measurements were carried out by scanning from a low potential limit where no faradic reaction occurs. The impedance measurements in each ionic liquid were performed at the formal potential calculated from the $\mathrm{CV}$ at the lowest scan rate. Each impedance spectrum was recorded from $10^{5}$ to $0.1 \mathrm{~Hz}$, and all the spectra were analyzed by fitting the parameters of the Randles equivalent circuit to the measured spectra.

Because of difficulty to achieve an exact concentration of Cc, for the determination of the diffusion coefficient, we use a combined measurement based on steady state and transient $\mathrm{CV}$ as explained in the text allowing a simultaneous determinations of the diffusion coefficient $D$ and initial concentration of $C^{\circ}$. For the experiment as function of the scan rate, the initial concentration was recalculated from the value of $D$ and peak current $I_{p}$ at low scan rates.

For experiments concerning the determination of the diffusion coefficient, $C^{\circ}$ and $D$ were determined simultaneously because of the difficulties of solubilizing $\mathrm{Cc}$ in the short chain RTILs. For example in typical experiments, in EMITFSI, the derived concentration is $3.5 \times 10^{-3} \mathrm{~mol} \mathrm{~L}^{-1}$, nearly one quarter of the nominal value (around $15 \times 10^{-3} \mathrm{~mol} \mathrm{~L}^{-1}$ ), while in BMIFTSI the concentration is $7.7 \times 10^{-3} \mathrm{~mol} \mathrm{~L}^{-1}$. For OMITFSI, because of the low value of $D$ in this RTIL, it was necessary to increase the initial concentration in OMITFSI to around $100 \mathrm{mM}$ in order to determine the diffusion coefficient accurately. The calculated concentration is $102.3 \mathrm{mM}$ in OMITFSI.

\section{Acknowledgements}

We thank the CNRS and the three Universities and school (ENSParis/Rennes 1/Xiamen/Wuhan) joint laboratory LIA NanoBioCatChem for financial support. In Xiamen, this work was supported by the Natural Science Foundation of China (Nos. 22072123, 21533006, 21972119, 21673193) and the 111 Project (Nos. B08027, B17027). We sincerely thank Prof. Christian Amatore for his valuable discussions and advices.

Keywords: Charge transfer kinetics $\cdot \mathrm{Ag}(111)$ single crystal • Ionic liquid • Interfacial charge transfer $•$ Alkyl side chain length

[1] a) M. Armand, F. Endres, D. R. MacFarlane, H. Ohno, B. Scrosati, Nat. Mater. 2009, 8, 621-629; b) L. E. Barrosse-Antle, A. M. Bond, R. G. Compton, A. M. O'Mahony, E. I. Rogers, D. S. Silvester, Chem. Asian. J. 2010, 5, 202-230; c) M. C. Buzzeo, R. G. Evans, R. G. Compton, ChemPhysChem 2004, 5, 1106-1120; d) M. V. Fedorov, A. A. Kornyshev, Chem. Rev. 2014, 114, 2978-3036; e) P. Hapiot, C. Lagrost, Chem. Rev. 2008, 108, 2238-2264; f) R. Hayes, G. G. Warr, R. Atkin, Chem. Rev. 2015, 115, 6357-6426; g) D. R. MacFarlane, J. M. Pringle, P. C. Howlett, M. Forsyth, Phys. Chem. Chem. Phys. 2010, 12, 1659-1669; h) X. Mao, P. Brown, C Cervinka, G. Hazell, H. Li, Y. Ren, D. Chen, R. Atkin, J. Eastoe, I. Grillo, A. A. H. Padua, M. F. Costa Gomes, T. A. Hatton, Nat. Mater. 2019, 18, 1350-1357; i) H. Ohno, Electrochemical aspects of ionic liquids, John Wiley \& Sons, Inc, Hoboken, 2011; j) Y. Z. Su, Y. C. Fu, Y. M. Wei, J. W. Yan, B. W. Mao, ChemPhysChem 2010, 11, 2764-2778; k) H. T. Liu, Y. Liu and J. H. Li, PCCP, 2010, 12, 1685-1697; I) D. E. Khoshtariya, T. D. Dolidze, R. van Eldik, Chem. Eur. J. 2009, 15, 5254-5262; m) V. A. Nikitina, A. V. Rudnev, G. A. Tsirlina, T. Wandlowski, J. Phys. Chem. C 2014, 118, 15970-15977; n) N. E. Martínez-Romero, R. Aguilar-Sánchez, Y. C. Fu, M. Homberger, U. Simon, Electrochim. Acta 2017, 231 44-52.

[2] a) L. G. Lin, Y. Wang, J. W. Yan, Y. Z. Yuan, J. Xiang, B. W. Mao, Electrochem. Commun. 2003, 5, 995-999; b) M. Gnahm, C. Berger, M. Arkhipova, H. Kunkel, T. Pajkossy, G. Maas, D. M. Kolb, Phys. Chem. Chem. Phys. 2012, 14, 10647-10652; c) M. Gnahm, T. Pajkossy, D. M. Kolb, Electrochim Acta 2010, 55, 6212 6217 ; d) Y. Z. Su, J. W. Yan, M. G. Li, Z. X. Xie, B. W. Mao, Z. Q. Tian, Z. Phys. Chem. 2012, 226, 979-994.

[3] a) M. Gnahm, C. Muller, R. Repanszki, T. Pajkossy, D. M. Kolb, Phys. Chem. Chem. Phys. 2011, 13, 11627-11633; b) C. Muller, S Vesztergom, T. Pajkossy, T. Jacob, J. Electroanal. Chem. 2015, 737, 218-225; c) Y. Z. Su, J. W. Yan, M. G. Li, M. Zhang, B. W. Mao, J. Phys. Chem. C 2013, 117, 205-212; d) J. Peng, M. G. Li, L. Q. Xie, J. W. Yan, B. W. Mao, J. Electrochem. 2016, 22, 596601.

[4] a) Y. C. Fu, A. V. Rudnev, Curr. Opin. Electroche. 2017, 1, 59-65; b) X. Zhang, Y. X. Zhong, J. W. Yan, Y. Z. Su, M. Zhang, B. W. Mao, Chem. Commun. 2012, 48, 582-584; c) Y. X. Zhong, J. W. Yan, M. G. Li, X. Zhang, D. W. He, B. W. Mao, J. Am. Chem. Soc. 2014, 136, 14682-14685; d) M. G. Li, L. Chen, Y. X. Zhong, Z. B. Chen, J. W. Yan, B. W. Mao, Electrochim Acta 2016, 197, 282289.

[5] a) M. V. Fedorov, N. Georgi, A. A. Kornyshev, Electrochem. Commun. 2010, 12, 296-299; b) R. M. Lynden-Bell, A. I. Frolov, M. V. Fedorov, Phys. Chem. Chem. Phys. 2012, 14, 2693-2701; c) C. Merlet, D. T. Limmer, M. Salanne, R. van Roij, P. A. Madden, D. Chandler, B. Rotenberg, J. Phys. Chem. C 2014, 118, 1829118298.

[6] M. Z. Bazant, B. D. Storey, A. A. Kornyshev, Phys. Rev. Lett. 2011, 106, 046102

[7] A. A. Kornyshev, L. P. Yang, Electrochem. Commun. 2014, 48, 173-177.

[8] a) J. M. Savéant, in Elements of Molecular and Biomolecular Electrochemistry, John Wiley \& Sons, Inc., Hoboken, 2006, pp. 481-485; b) M. J. Weaver, Chem. Rev. 1992, 92, 463-480.

[9] a) C. L. Bentley, J. Z. Li, A. M. Bond, J. Zhang, J. Phys. Chem. C 2016, 120, 16516-16525; b) W. R. Fawcett, A. Gaal, D. Misicak, J. Electroanal. Chem. 2011, 660, 230-233; c) N. Fietkau, A. D. Clegg R. G. Evans, C. Villagran, C. Hardacre, R. G. Compton, ChemPhysChem 2006, 7, 1041-1045; d) C. Lagrost, D. Carrié, M. Vaultier, P. Hapiot, J. Phys. Chem. A 2003, 107, 745-752; e) C. Lagrost, L. Preda, E. Volanschi, P. Hapiot, J. Electroanal. Chem. 2005, 585, 1-7; f) R. M. Lynden-Bell, Electrochem. Commun. 2007 9, 1857-1861.

W. R. Fawcett, J. Solid. State. Electr. 2011, 15, 1347-1358. 
[11] J. D. L. Holloway, W. E. Geiger, J. Am. Chem. Soc. 1979, 101, 2038-2044.

[12] F. Zhen, L. Percevault, L. Paquin, E. Limanton, C. Lagrost, P. Hapiot, J. Phys. Chem. B 2020, 124, 1025-1032.

[13] a) C. P. Andrieux, P. Hapiot, J. M. Saveant, Chem. Rev. 1990, 90, 723-738; b) C. P. Andrieux, D. Garreau, P. Hapiot, J. Pinson, J. M. Saveant, J. Electroanal. Chem. 1988, 243, 321-335; c) J. C. Imbeaux, J. M. Savéant, J. Elecanalyt. Chem. Inter. Electrochem. 1970, 28, 325-338.

[14] a) J. E. Baur, R. M. Wightman, J. Electroanal. Chem. 1991, 305, 73-81; b) Y. J. Wang, E. I. Rogers, R. G. Compton, J. Electroanal. Chem. 2010, 648, 15-19; c) K. Aoki, J. Osteryoung, J. Electroanal. Chem. 1984, 160, 335-339.

[15] a) S. K. Sukardi, J. Zhang, I. Burgar, M. D. Horne, A. F. Hollenkamp, D. R. MacFarlane, A. M. Bond, Electrochem Commun. 2008, 10, 250-254; b) M. J. Shiddiky, A. A. Torriero, C. Zhao, I. Burgar, G. Kennedy, A. M. Bond, J. Am. Chem. Soc. 2009, 131, 7976-7989.

[16] a) J. M. Savéant, J. Electroanal. Chem. 1975, 65, 57-66; b) J. C. Imbeaux, J. M. Savéant, J. Elecanalyt. Chem. Inter. Electrochem. 1973, 44, 169-187.

[17] K. Nakamura, T. Shikata, ChemPhysChem 2010, 11, 285-294.

[18] a) V. O. Santos, Jr., M. B. Alves, M. S. Carvalho, P. A. Suarez, J. C. Rubim, J. Phys. Chem. B 2006, 110, 20379-20385; b) J. C.

Rubim, F. A. Trindade, M. A. Gelesky, R. F. Aroca, J. Dupont, J. Phys. Chem. C 2008, 112, 19670-19675; c) Y. Liu, Y. X. Yuan, X. R. Wang, N. Zhang, M. M. Xu, J. L. Yao, R. A. Gu, J. Electroanal. Chem. 2014, 728, 10-17.

[19] a) L. V. Protsailo, W. R. Fawcett, Electrochim Acta 2000, 45, 3497 3505; b) D. J. Gavaghan, S. W. Feldberg, J. Electroanal. Chem. 2000, 491, 103-110 
WILEY-VCH

\section{Entry for the Table of Contents}

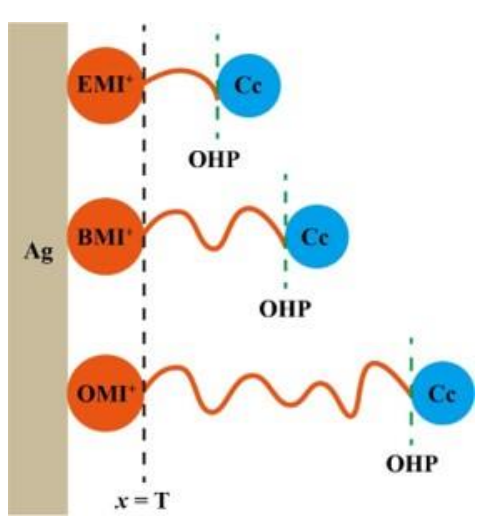

The effect of electrode/ionic liquid interfaces on the charge transfer kinetics of the oxidation of chromocene at a single crystal Ag(111) electrode was studied. The variations of standard charge transfer rate constants with cation alkyl chain lengths demonstrate that wellorganized electrode/RTIL interfaces behave as a sort of self-assembled monolayer that controls the charge transfer kinetics with an exponential tunneling coefficient of $5.51 \mathrm{~nm}^{-1}$.

Miangang Li, Shuai Liu, Liqiang Xie, Jiawei Yan*, Corinne Lagrost, Shuai Wang, Guang Feng, Philippe Hapiot*, Bingwei Mao* 